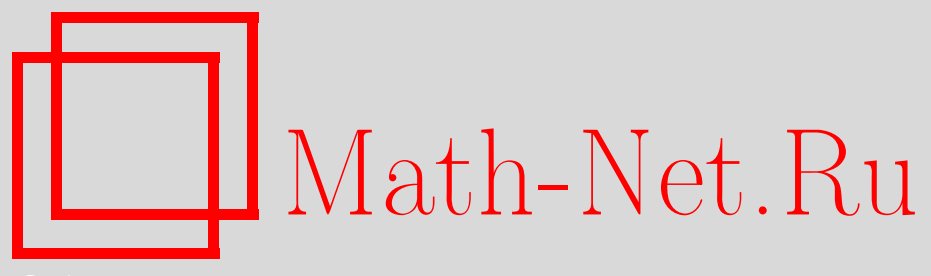

В. В. Рыжиков, О самоприсоединениях коммутативных действий с инвариантной мерой, Матем. заметки, 2008, том 83, выпуск 5, 792-795

DOI: https://doi.org/10.4213/mzm4726

Использование Общероссийского математического портала Math-Net.Ru подразумевает, что вы прочитали и согласны с пользовательским соглашением http://www . mathnet.ru/rus/agreement

Параметры загрузки:

IP : 107.22 .136 .117

26 апреля 2023 г., 15:04:49

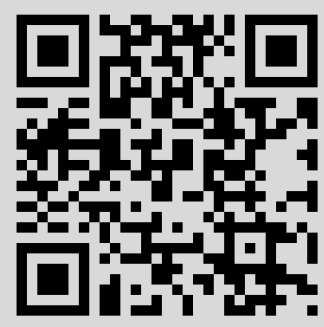




\section{О самоприсоединениях коммутативных действий с инвариантной мерой}

\section{В. В. Рыжиков}

Для изучения динамической системы с инвариантной мерой представляет интерес ее взаимодействие с другими системами и ее расположение в полугруппе марковских операторов. Вопросы о централизаторе действия, о структуре его факторов - частный случай этой общей задачи. Полезным инструментом в ее исследовании являются джойнинги. Преобразования с минимальными самоприсоединениями (self-joinings) введены Рудольфом в эргодическую теорию как средство для конструирования действий с необычными разнообразными свойствами. У преобразований с минимальными самоприсоединениями, в частности, нет факторов, а их централизатор тривиален. Это стимулировало изучение более общего класса так называемых простых систем (см., например, [1]-[3]).

Пусть $T: X \rightarrow X$ - сохраняющее меру обратимое преобразование пространства с мерой $(X, \mathscr{B}, \mu), \mu(X)=1$. Мера $\nu$ на $X_{(1)} \times \cdots \times X_{(n)}$ называется самоприсоединением порядка $n$, если она $T_{(1)} \times \cdots \times T_{(n)}$-инвариантна и ее проекции на $X_{(i)}$ равны $\mu$. В определении самоприсоединения группового действия $\left\{T_{g}: g \in G\right\}$ предполагается инвариантность меры $\nu$ относительно $T_{g} \times \cdots \times T_{g}$ для всех $g$.

Образ меры $\mu$ при отображении $\varphi_{S}: X \rightarrow X \times X$, где $\varphi_{S}(x)=(x, S(x))$, обозначается $\Delta_{S}$ (сдвиг диагональной меры на $\left.X \times X\right)$. Заметим, что $\Delta_{S}$ определена равенством $\Delta_{S}(A \times B)=\mu(S A \cap B)$. Если автоморфизм $S$ коммутирует с действием $\left\{T_{g}: g \in G\right\}$, то $\Delta_{S}$ является самоприсоединением порядка 2. Действие $\left\{T_{g}\right\}$ называется 2-простыз, если все эргодические самоприсоединения порядка 2 имеют вид $\Delta_{S}$ или являются мерой $\mu \times \mu$.

Для 2-простой системы крайние точки в полугруппе всех коммутирующих с системой марковских операторов состоят из коммутирующих с ней автоморфизмов и оператора $\Theta$ - ортопроекции на пространство констант в $L_{2}(\mu)$.

Действие $\left\{T_{g}\right\}$ называется $n$-nростым, если любое его эргодическое самоприсоединение $\nu$ порядка $n$ или совпадает с мерой $\mu^{n}$, или одна из проекций меры $\nu$ на некоторую двумерную грань в кубе $X_{1} \times \cdots \times X_{n}$ имеет вид $\Delta_{S}$.

Исследование самоприсоединений высоких порядков тесно связано с проблемой Рохлина о кратном перемешивании. В классе автоморфизмов с минимальным марковским централизатором существование нетривиального самоприсоединения порядка 3 влечет решение этой знаменитой проблемы (см. [3]), а для перемешивающего автоморфизма положительного локального ранга дает как решение проблемы Рохлина так и решение ослабленной проблемы Банаха об абсолютно непрерывном спектре конечной кратности (см. ссылки в [4]). По этим и другим причинам вопрос о совпадении 2-простоты коммутативного действия со свойством простоты высоких порядков является центральным в теории самоприсоединений.

Действие прямой суммы конечных групп изучалось в спектральной теории Степиным [5]. Рост интереса к динамическим системам с неклассическим временем - тенденция последних лет (см. обзор [6]). Групповые действия успешно применяются для изучения индивидуальных автоморфизмов, входящих в эти действия ([2], [7], [8]).

Настоящая заметка посвящена обобщению результатов [3] о 2-простых динамических системах с классическим временем на коммутативные групповые действия. В частности, нами будет установлено, что построенные в [7] 2-простые действия групп $\mathbb{Z}^{n} \times \mathbb{R}^{m}$ обладают кратым перемешиванием и свойством простоты всех порядков.

Работа выполнена при финансовой поддержке программы "Ведущие научные школы" (грант № НШ-6849.2006.1). 
1. Действия несчетных групп. Будем говорить, что действие $\left\{T_{g}\right\}$ группы $G$ обладает тотальным слабым перемешиванием, если каждое его бесконечное поддействие содержит перемешивающую последовательность. Напомним, что последовательность $T_{g_{i}}$ называется перемешивающей, если $T_{g_{i}} \rightarrow \Theta$. Если последнее выполнено для всех последовательностей $g_{i} \rightarrow \infty$, то действие $\left\{T_{g}\right\}$ называется перемешивающим.

Теорема 1. Пусть $G$-локально компактная несчетная абелева группа. Тогда 2-простое тотально слабо перемешивающее $G$-действие является простым всех порядков.

СледствиЕ. Несчетное 2-простое перемешивающее действие перемешивает с любой кратностью.

ЗАмечАниЕ. Примеры $\mathbb{Z}^{n} \times \mathbb{R}^{m}$-действий, построенные в работе [7], удовлетворяют условию теоремы.

ДоКАЗАТЕЛЬСТво тЕОРЕмЫ 1 . Пусть эргодическое самоприсоединение $\nu$ порядка 3 обладает свойством: проекции $\nu$ на двумерные грани в произведении $X \times X \times X$ совпадают с $\mu \times \mu$. Нужно установить, что $\nu=\mu \times \mu \times \mu$. Этим простота порядка 3 будет доказана. Предположим, что самоприсоединение $\nu$ отлично от $\mu \times \mu \times \mu$.

Будем писать $g \sim g^{\prime}$, если найдется $R=R_{g, g^{\prime}}\left(R \in C\left(\left\{T_{g}\right\}\right)\right)$ такой, что

$$
\left(I \otimes I \otimes T_{g^{-1}} T_{g^{\prime}}\right) \nu=(R \otimes I \otimes I) \nu
$$

( $I$ - тождественный оператор в $\left.L_{2}(\mu)\right)$. Очевидно, что отношение $\sim$ является эквивалентностью, а множество

$$
H_{\nu}=\{h: h \sim e\}
$$

является подгруппой $G$.

Лемма. Число классов эквивалентности не более, чем счетно.

Лемма является непосредственным следствием леммы 1 из [3], которая (как и используемая в [3] теорема 1 из [1]) верна для действий любых групп.

Из леммы непосредственно вытекает, что факторгруппа $G / H_{\nu}$ не более чем счетна, поэтому $\left|H_{\nu}\right|=\infty$. Так как рассматриваемое действие обладает тотальным слабым перемешиванием, действие группы $H_{\nu}$ содержит перемешивающую последовательность $\left\{h_{i}\right\}$, т.е.

$$
T_{h_{i}} \rightarrow \Theta, \quad h_{i} \in H_{\nu} .
$$

Из определения группы $H_{\nu}$ вытекает, что для каждого $i$ найдется автоморфизм $R_{i}$ такой, что $\left(R_{i} \otimes I \otimes T_{h_{i}}\right) \nu=\nu$. Это равносильно

$$
\int f_{1} \otimes f_{2} \otimes f_{3} d \nu=\int R_{i} f_{1} \otimes f_{2} \otimes T_{h_{i}} f_{3} d \nu
$$

(здесь $R_{i}$ обозначают операторы в $L_{2}(X, \mathscr{B}, \mu)$, индуцированные одноименными автоморфизмами). Пусть марковский оператор $R$ - предельная точка для последовательности операторов $\left\{R_{i}\right\}$. Воспользовавшись тем, что присоединение $\nu$ обладает попарной независимостью, получаем

$$
\begin{gathered}
\int f_{1} \otimes f_{2} \otimes f_{3} d \nu=\int R f_{1} \otimes f_{2} \otimes \Theta f_{3} d \nu=\int f_{1} d \mu \int f_{2} d \mu \int f_{3} d \mu, \\
\nu=\mu \times \mu \times \mu .
\end{gathered}
$$

Случай порядка $n>3$ рассматривается аналогично (впрочем, из известного результата Дж. Кинга вытекает, что простота порядка 4 влечет за собой простоту всех порядков). 


\section{2. Действия счетных групп.}

Теорема 2. Пусть 2-простое действие $\left\{T_{g}\right\}$ счетной абелевой группы обладает тотальным слабым перемешиванием, но не является перемешивающим. Тогда $\left\{T_{g}\right\}$ обладает простотой всех порядков.

СлЕДСтвиЕ. Любое перемешивающее действие (включая некоммутативные), коммутирующее с таким действием $\left\{T_{g}\right\}$, обладает свойством кратного перемешивания всех порядков.

ЗамечАниЕ. А. И. Даниленко и А. дель Юнко сообщили автору, что соответствующие пары из перемешивающего и неперемешивающего действий можно построить методом стохастических конструкций.

ДОКАЗАТЕЛЬСТВо ТЕОРЕМЫ 2. ПредПоЛожим, что эргодическое самоприсоединение $\nu$ трех копий рассматриваемого действия отлично от меры $\mu \times \mu \times \mu$. Рассмотрим группу $H_{\nu}$ всех таких $h \in G$, когда найдется автоморфизм $R$ такой, что

$$
\left(I \otimes I \otimes T_{h}\right) \nu=(R \otimes I \otimes I) \nu .
$$

В случае бесконечной группы $H_{\nu}$, повторяя рассуждения при доказательстве теоремы 1 , получим $\nu=\mu \times \mu \times \mu$.

Таким образом, осталось рассмотреть случай, когда группа $H_{\nu}$ конечна. Пусть $h$ не принадлежит $H_{\nu}$. Тогда для оператора $J: L_{2}(\mu) \rightarrow L_{2}(\mu) \otimes L_{2}(\mu)$, связанного с мерой мерой $\nu$ соотношением

$$
\int_{X \times X \times X} f_{1} \otimes f_{2} \otimes f_{3} d \nu=\int_{X \times X}\left(J f_{1}\right) f_{2} \otimes f_{3} d \mu \times \mu
$$

выполнено

$$
\Theta=J^{*}\left(I \otimes T_{h}\right) J .
$$

Этот факт непосредственно вытекает из результатов [1], [3].

Пусть $h_{i}-$ последовательность различных элементов группы $G$, для которой выполнено условие

$$
T_{h_{i}} \rightarrow P^{*} P \neq \Theta,
$$

где $h_{i}=g_{i}^{-1} g_{j(i)}, g_{i}$ - некоторая последовательность такая, что

$$
T_{g_{i}} \rightarrow P \neq \Theta .
$$

Нужная последовательность $g_{i}$ всегда найдется, так как исходное действие не обладает свойством перемешивания.

Так как все $h_{i}$, кроме конечного числа, не принадлежат группе $H_{\nu}$, получим

$$
\Theta=J^{*}\left(I \otimes T_{h}\right) J \rightarrow J^{*}\left(I \otimes P^{*} P\right) J .
$$

Следовательно,

$$
\begin{gathered}
((I \otimes P) J)^{*}(I \otimes P) J=\Theta, \\
(I \otimes P) J=\Theta^{\prime}
\end{gathered}
$$

где оператор $\Theta^{\prime}: L_{2}(\mu) \rightarrow L_{2}(\mu) \otimes L_{2}(\mu)$ отвечает мере $\mu \times(\mu \times \mu)$, его образ - константы. Важным фактом является неразложимость сплетения $\Theta^{\prime}$, которая эквивалентна эргодичности меры $\mu \times(\mu \times \mu))$.

Рассматриваемое действие $\left\{T_{g}\right\}$ является 2-простым, поэтому имеет место разложение оператора $P$ в виде

$$
P=a \int S_{\alpha} d \eta(\alpha)+(1-\alpha) \Theta, \quad a>0,
$$


где $\eta$ - некоторая мера на централизаторе действия. Из неразложимости оператора $\Theta^{\prime}$ вытекает, что для почти всех $\alpha$ (нам достаточно одного $\alpha$ ) имеет место равенство $\Theta^{\prime}=$ $\left(I \otimes S_{\alpha}\right) J$. В силу обратимости оператора $I \otimes S_{\alpha}$ получаем $J=\Theta^{\prime}$, что равносильно равенству $\nu=\mu \times \mu \times \mu$.

Доказательство тривиальности самоприсоединений порядка $n>3$ аналогично.

Свойство коммутативности действий существенно для наших целей, так как имеются примеры 2-простых некоммутативных действий, не обладающих простотой порядка 3 (см. ссылки в [3]). Вероятно, изложенные результаты могут быть перенесены на квазипростые коммутативные действия (см. [8], [9]).

В связи с результатами работы [3] возникает вопрос: влечет ли свойство 2-простоты свойство простоты всех порядков (и, следовательно, кратное перемешивание всех порядков) для коммутативных действий, обладающих перемешиванием кратности 1 (кратности 2)?

\section{СПИСОК ЦИТИРОВАННОЙ ЛИТЕРАТУРЫ}

[1] E. Glasner, B. Host, D. Rudolph, Israel J. Math., 78:1 (1992), 131-142. [2] A. del Junco, Israel J. Math., 104 (1998), 301-320. [3] V. V. Ryzhikov, J. Dynam. Control Systems, 3:1 (1997), 111-127. [4] В. В. Рыжиков, Функи. анализ и его прил., 34:1 (2000), 90-93. [5] А. М. Степин, Докл. АН СССР, 169:4 (1966), 773-776. [6] A. I. Danilenko, Geometry an Dynamics of Groups and Spaces, Progr. Math., 265, 2007, 236-263. [7] A. I. Danilenko, C. Silva, Ann. Inst. H. Poincaré Probab. Statist., 43:4 (2007), 375-398. [8] A. I. Danilenko, J. Anal. Math., 102:1 (2007), 77-117. [9] В. В. Рыжиков, ЖК.-П. Тувено, Функи. анализ и его прил., 40:3 (2006), 85-89.

\section{В. В. Рыжиков}

Поступило

Московский государственный 31.10 .2007

университет им. М. В. Ломоносова

E-mail: vryzh@mail.ru 